\title{
The effect of the ultrasonic irradiation on clearance in dialyzer-model
}

\author{
Nobuhide Tatsumoto, ${ }^{*}$ Nagamasa Kawano, ${ }^{*}$ Mitsuru Tsuda, ${ }^{*}$ Shouji Harada, ${ }^{* *}$ \\ and Shigetada Fujii* \\ *Medical College of Oita, \\ Hazama-cho, Oita-gun, Oita, 879-56 Japan \\ **Faculty of Science, Hiroshima University, \\ Higashisenda-machi, Naka-ku, Hiroshima, 730 Japan
}

(Received 21'April 1988)

\begin{abstract}
In the dialysis experiment where an artificial kidney was used as a model, and an aqueous solution of creatinine was used instead of blood, the clearance, dialysis efficiency, was improved remarkably by ultrasonic irradiation. The clearance increased with the ultrasonic level and reached the maximum value at a certain ultrasonic level. The maximum value of clearance was about 1.5 times as high as that without ultrasonic irradiation. The dialysis in this dialyzer model was treated with a simple transfer theory on the inside of the dialyzer tube, in the tube wall, and in the outer region of the tube considering the diffusion resistance respectively. The derived relation of clearance to the volume flow was compared with the experimental results. It has been deduced that the higher values of the clearance were due to the removal of the outer concentration boundary layer by ultrasonic cavitation and microstream. Thus, the predominated resistance was considered to be mainly due to that in the tube wall in this situation, and the value of diffusion coefficient was about $7.7 \times 10^{-7} \mathrm{~cm}^{2} / \mathrm{s}$.
\end{abstract}

PACS number: 43. 35. Ty, 43. 80. Vj

\section{INTRODUCTION}

One of the most important functions of the dialyzer is the elimination of waste in blood by filtration. ${ }^{1)}$ It is always carried out naturally in healthy persons, but not in patients of functional disease in the kidney. The artificial elimination in the patients, therefore, has to be done by using the dialyzer at regular intervals, during which they must keep quiet. Then, it is quite necessary to reduce the eliminating time. The authors made a preliminary test to increase the efficiency of dialysis by irradiating ultrasound on the dialyzer model, because the following ultrasonic mechanical actions are expected to increase clearance; that is, the repetitional sound pressure increases the relative velocity of particles and induces the ultrasonic straight flow, microstream, and cavitation which effectively stir the inner or outer solutions of the dialysis membrane. A remarkable enhancement of the efficiency of dialysis was found in this test. The reports on the effect of ultrasound on the dialysis efficiency, however, are hardly found. The purpose of this study is, therefore, to clarify the causes of the ultrasonic effect on the dialysis efficiency.

\section{THEORETICAL BACKGROUND}

The concentration of solute, waste molecule, denoted $C$, in the solution, which flows inside a hollow fiber tube for dialysis as shown in Fig. 1, may be regarded as a function of time, $t$. The concentration per unit distance, therefore, may be represented by $C(t)$ at a certain position in the flow direction. The change with $t$ in the concentration of solute inside the hollow fiber tube, hereinafter abbreviated as the tube, can be considered to be 


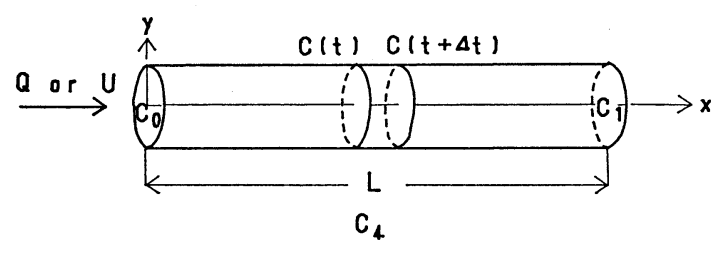

Fig. 1 The schematic diagram of the hollow fiber tube. $C_{4}$ is the solute concentration in the bulk of the dialyzer.

proportional to the difference between the solute concentrations in the inside and the outside of the tube, and if that in the outside of the tube is much smaller than that in the inside, the following equation is given;

$$
-d C / d t=K C(t),
$$

where $K$ is a rate constant of the solute which passed through the tube membrane. Hence, the solute concentration at the inlet of the tube, $t=0$, is defined as $C_{0}$, and then the solute concentration at the outlet, $C_{1}$, is given by

$$
C_{1}=C_{0} \exp (-K V / Q) \text {. }
$$

where $V$ is the total effective volume of the inside of the tube, $Q$ is the volume flow of the solution per unit time. The solute molecule may pass through three layers, i.e. the dialysis membrane and the inner and outer concentration boundary layers on the dialysis membrane ${ }^{1)}$ as shown in Fig. 2. These three layers can be safely regarded approximately as a plane, where the layers are much thinner than the radius of the tube. Hence, the resistance of the solute transport through the tube membrane, $R$, is represented by the diffusion theory as follows;

$$
R=R_{\text {in }}+R_{\mathrm{M}}+R_{\text {out }},
$$

where $R_{\mathrm{in}}, R_{\mathrm{M}}$, and $R_{\text {out }}$ are the solute transport resistances in the inner concentration boundary layer, membrane, and outer concentration boundary layer respectively. The permeabilities which are the reciprocal of $R$, therefore, also consist of the following three terms;

$$
R=\left(1 / k_{\text {in }}\right)+\left(1 / k_{\mathrm{M}}\right)+\left(1 / k_{\text {out }}\right),
$$

where $k_{\text {in }}, k_{\mathrm{M}}$, and $k_{\text {out }}$ are the permeabilities in the inner concentration boundary layer, membrane, and the outer concentration boundary layer respectively. These permeabilities are related with the rate constant $K$, as the mol numbers of the solute which passed through are equal to each other in the three

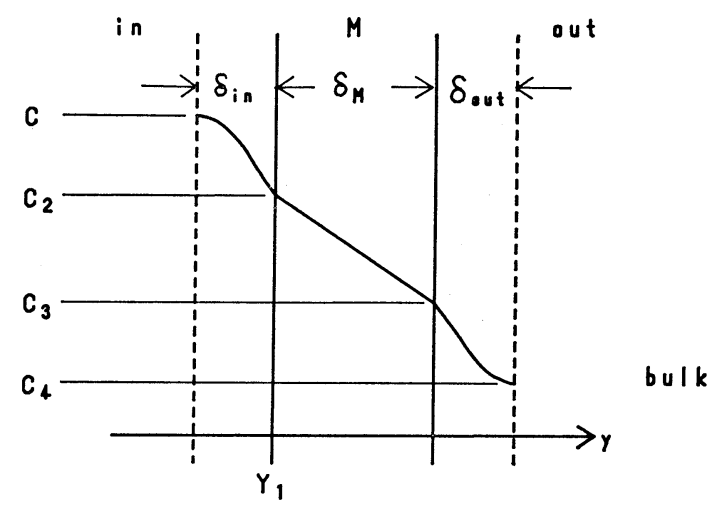

Fig. 2 The schematic diagram of solute concentration gradient in the neighborhood of dialysis membrane.

layers. Therefore, the following equation is given;

$$
\begin{aligned}
B & =V(\partial C / \partial t) \\
& =A_{\text {in }} k_{\text {in }}\left(C-C_{2}\right) \\
& =A_{\mathrm{M}} k_{\mathrm{M}}\left(C_{2}-C_{3}\right) \\
& =A_{\text {out }} k_{\text {out }}\left(C_{3}-C_{4}\right) \\
& =V K\left(C-C_{4}\right),
\end{aligned}
$$

where $A_{\mathrm{M}}, A_{\text {in }}$, and $A_{\text {out }}$ are effective areas of the tube membrane, the inside and outside of the membrane, and $C_{2}, C_{3}$, and $C_{4}$ are the concentrations on the inner and outer surface of the membrane, and in the bulk of the dialyzer as shown in Fig. 2 respectively. Therefore, the following equation is given;

$$
\begin{aligned}
& B\left(\frac{1}{k_{\text {in }} A_{\text {in }}}+\frac{1}{k_{\mathrm{M}} A_{\mathrm{M}}}+\frac{1}{k_{\text {out }} A_{\text {out }}}\right) \\
& \quad=C-C_{4}=\frac{B}{K V},
\end{aligned}
$$

where $A_{\text {in }}, A_{\mathrm{M}}$, and $A_{\text {out }}$ are represented by

$$
\begin{aligned}
& A_{\text {in }}=2 \pi Y_{1} L\left\{1-\left(\delta_{\text {in }} / Y_{1}\right)\right\}, \\
& A_{\mathrm{M}}=2 \pi Y_{1} L, \\
& A_{\text {out }}=2 \pi Y_{1} L\left\{1-\left(\delta_{\mathrm{M}} / Y_{1}\right)\right\},
\end{aligned}
$$

respectively. Assuming $\delta_{\text {in }} / Y_{1}$ and $\delta_{\text {out }} / Y_{1}<1$, $A_{\text {in }}=A_{\mathrm{M}}=A_{\text {out }}$, where $\delta_{\mathrm{M}}, \delta_{\text {in }}$, and $\delta_{\text {out }}$ are the thickness of the tube membrane, the inside and outside of the membrane respectively, and $Y_{1}$ is an inner radius of the tube, and $L$ is an effective length of the tube. Hence,

$$
\left(1 / k_{\text {in }}\right)+\left(1 / k_{\mathrm{M}}\right)+\left(1 / k_{\text {out }}\right)=\left(2 / K Y_{1}\right),
$$

and then since $Y_{1}$ is constant, the following relation 


\section{N. TATSUMOTO et al.: ULTRASONIC EFFECT ON CLEARANCE IN DIALYZER-MODEL}

is given;

$$
\sum\left(1 / k_{\mathrm{i}}\right) \propto 1 / K .
$$

Both of clearance, $C_{\mathrm{L}}$, and dialysance, $D_{\mathrm{L}}$, are the parameters which represent the dialysis efficiency per unit time, and these are defined as follows; $;{ }^{1,2}$

$$
\begin{aligned}
& C_{\mathrm{L}}=Q\left(C_{0}-C_{1}\right) / C_{0}, \\
& D_{\mathrm{L}}=Q\left(C_{0}-C_{1}\right) /\left(C_{0}-C_{4}\right) .
\end{aligned}
$$

\section{APPARATUS AND EXPERIMENTAL}

The dialyzer model of hollow fiber kidney type consists of a bundle of hollow fiber tubes (1) set up in a $50 \mathrm{ml}$ beaker (2) and a screw (3) to stir the dialysate in the beaker as shown in Fig. 3. The following equipments are connected to the dialyzer; a small reservoir $(50 \mathrm{ml})(4)$ for the waste solution instead of blood; a pump (5) to supply the solution to the hollow fiber tube; a large reservoir (1 $l$ ) (6) for the dialysate; a pump (7) to circulate the dialysate; a pump (8) to circulate the thermostatted water (9) in the whole equipments. Poly-vinyl chloride tubes of $2 \mathrm{~mm}$ in an inner diameter (10), which were covered with polystyrene foam sheet (11) for the maintenance of a constant temperature, were used in the transports of the waste solution and dialysate through those equipments. A rotational speed of the pump (5) for the supply of the waste solution was controlled particularly by an outer DC stabilized supply. Since an electromotive force of a driving motor of the pump was kept below $\pm 0.1 \%$ in a fluctuation, which was observed with a digital volt meter, the fluctuation of rotational speed of the pump might be also assumed to be less than $\pm 0.1 \%$. On the other hand, the pump (7) controlled only by a bild-in DC stabilizer circulated the dialysate at the volume flow of $70( \pm 5 \%) \mathrm{ml} / \mathrm{min} . \quad 50 \mathrm{ml}$ dialysate, which was always preserved in a $50 \mathrm{ml}$ beaker (2) of the dialyzer, was stirred by the screw (3) jointed to a stepping motor (12). The stepping motor, which was driven by a driving amplifier with a crystal pulse oscillator, SMD-2, produced by Kairiku Denpa Co. Ltd., Hiroshima, was maximum 1,500 RPM in a rotational speed, $S_{v}$, and less than $\pm 0.1 \%$ in the fluctuation of $S_{\mathrm{v}}$. The hollow fiber tube, the product of Asahi Medical Co. Ltd. was made of copper ammonium rayon, $200 \mu \mathrm{m}$ in an inner diameter, and $11 \mu \mathrm{m}$ in a wall membrane thickness. The dialyzer was made of a bundle of

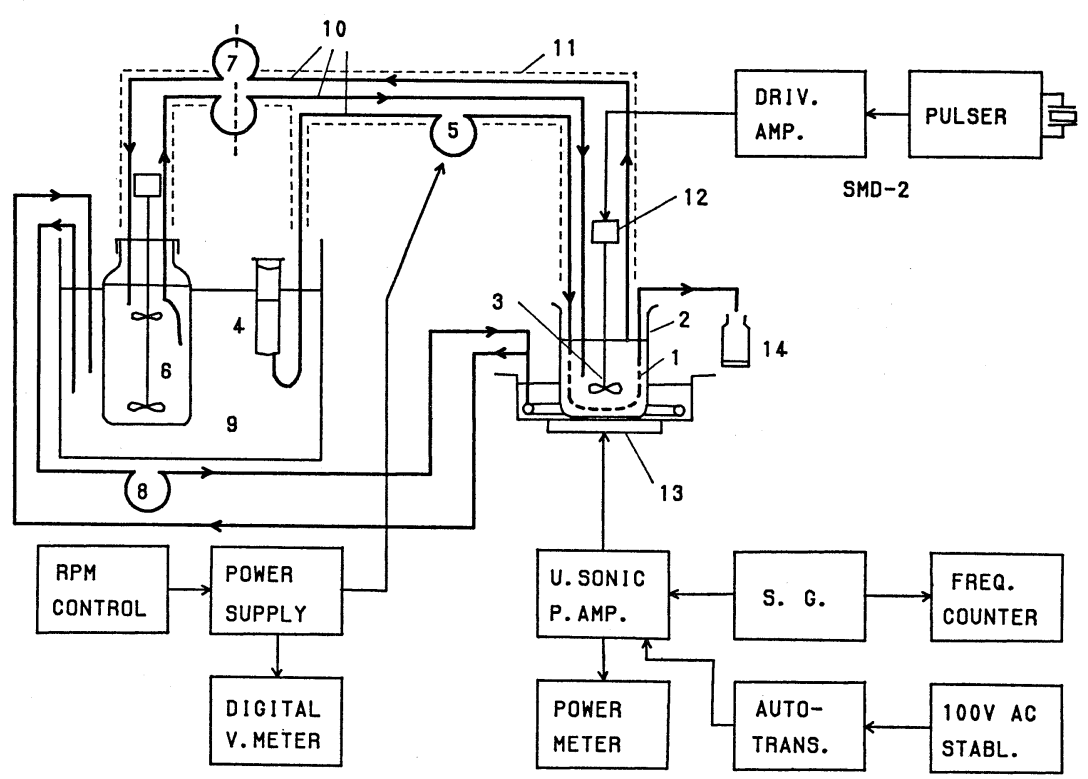

Fig. 3 Brock diagram of the dialyzer-model and ultrasonic apparatus. 1, bundle of hollow fiber tubes; 2, $50 \mathrm{ml}$ beaker; 3, screw; 4, small reservoir; 5, solution supplying pump; 6 , large reservoir; 7 , dialysate circulating pump; 8 , thermostatted water circulating pump; 9 , thermostatted water bath; 10 , poly-vinyl chloride tube; 11 , polystyrene foam sheet; 12 , stepping motor; 13 , ultrasonic transducer; 14 , sample tube. 
20 tubes and cut to an effective length, $L$, of 16 and $4 \mathrm{~cm}$. Those bundles were loosely fixed by a helically shaped vinyl-coated-copper wire, $0.5 \mathrm{~mm}$ in a diameter, where the helix of the wire was $6 \mathrm{~cm}$ in an inside diameter as shown in Fig. 4. The bundle of $L=16 \mathrm{~cm}$ was fixed particularly on the three PMMA, poly-methyl methacrylate, posts of $3 \mathrm{~mm}$ in diameter erected on the bottom of PMMA cap of $50 \mathrm{ml}$ beaker (2). Ultrasound was irradiated from the bottom of $50 \mathrm{ml}$ beaker (2) by an remodeled ultrasonic cleaner, Pet Cleaner 52, produced by Cho-onpa Kogyo Co. Ltd., Tachikawa, Tokyo. The frequency of ultrasound was $41 \pm 0.5 \mathrm{kHz}$ and controlled in $P_{\mathrm{a}}=0 \sim 30 \mathrm{~W}$ by the applied electric voltage, less than AC $100 \mathrm{~V}$, where $P_{\mathrm{a}}$ is an average electric power input to the transducer (13). The value of $P_{\text {a }}$ was measured by an analog electric power meter with the accuracy within $\pm 5 \%$ connected to input terminals of an output transformer. The stabilities of $P_{\mathrm{a}}$ in the experimental conditions were all within $\pm 5 \%$.

The $1.00 \times 10^{-2} \%$ aqueous solution of creatinine, $\mathrm{CH}_{3} \mathrm{NC}(: \mathrm{NH}) \mathrm{NHC}(\mathrm{O}) \mathrm{CH}_{2}$, molecular weight = 113.12 , which is one of the wastes, was used instead of blood. Ion-exchanged water was used as the dialysate in order to simplify the dialysis system. The concentration of creatinine was determined by the absorption measurement of ultraviolet light of wavelength $234 \mathrm{~nm}$. In a series of measurement of change in $C_{\mathrm{L}}$ with $Q$ at constant $S_{\mathrm{v}}$ and $P_{\mathrm{a}}$, an increase in $C_{4}$ was less than $2 \%$ of $C_{0}$ and the difference between values of $C_{\mathrm{L}}$ and $D_{\mathrm{L}}$ was extremely small, so that the dialysis efficiency will be expressed by the clearance, $C_{\mathrm{L}}$, hereinafter. The value of $Q$ was determined by the weight of the creatinine solution

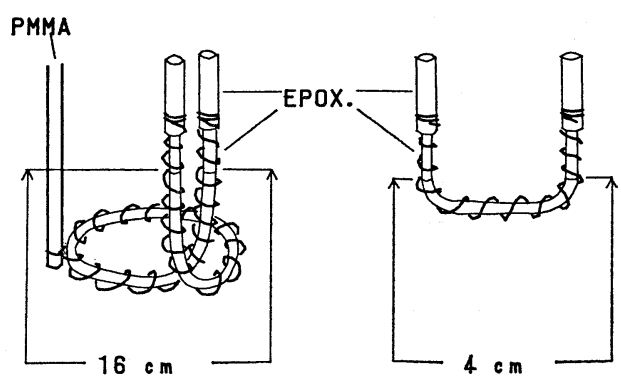

Fig. 4 The schemes of bundles of hollow fibers. EPOX.-parts molded by epoxy resin. collected in a sample tube (14) in Fig. 3 and its dropping time. The density of the creatinine solution, which was very diluted, was equal to that of water. A viscosity of the solution was measured with a rotational viscometer. All the measurements were made at $30^{\circ} \mathrm{C}$.

\section{RESULTS AND DISCUSSION}

4.1 Increases in Clearance by Ultrasonic Irradiation

The value of $C_{\mathrm{L}}$ in the hollow fiber tubes used in this experiment decreased with time and reached a minimum stationary value in about a week in the condition of $S_{\nabla}, P_{\mathrm{a}}$, and $Q=$ constant. The study on the decrease in $C_{\mathrm{L}}$ with time is very interesting as another subject. Therefore, the bundle of hollow fiber tubes, abbreviated again as tube hereinafter, soaked in water for a week was used in this experiment. The measurements of $C_{\mathrm{L}}$ were made mainly by using the bundle of the tubes of $L=16 \mathrm{~cm}$, because $C_{\mathrm{L}}$ changed largely with $Q$ and $S_{\mathrm{v}}$. The experimental values of $C_{\mathrm{L}}$ increased with $Q$ as shown in Fig. 5. The position of curve of $C_{\mathrm{L}}$ vs $Q$ went up with the increase of the rotational speed of screw, $S_{\mathrm{v}}$, which was proportional to the stirring speed of water, dialysate, without ultrasonic irradiation.

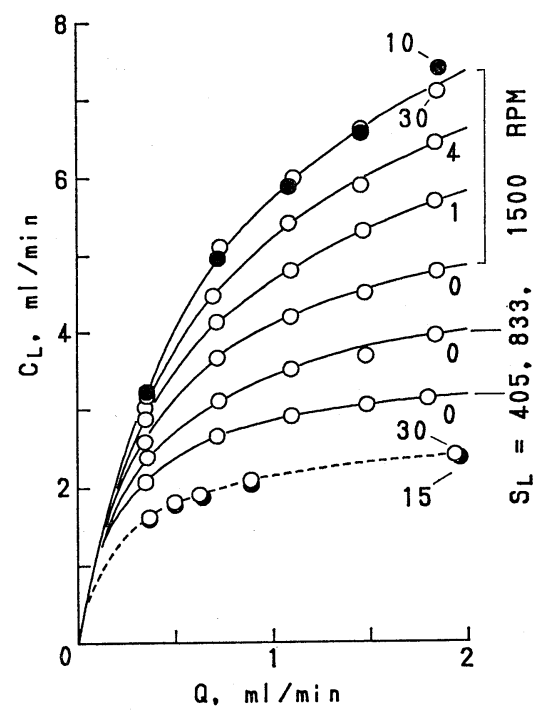

Fig. 5 The changes in $C_{\mathrm{L}}$ with $Q$ at various $S_{\vee}$ and $P_{\mathrm{a}}$. The numbers in this figure are $P_{\mathrm{a}}(\mathrm{W})$, and solid lines and a dushed line indicate the changes at $L=16$ and $4 \mathrm{~cm}$ $\left(S_{\nabla}=1,500 \mathrm{RPM}\right)$, respectively. 


\section{N. TATSUMOTO et al.: ULTRASONIC EFFECT ON CLEARANCE IN DIALYZER-MODEL}

When the ultrasound was irradiated, the position of curve went up more with the increase of the ultrasonic power, $P_{\mathrm{a}}$, and reached the maximum position at $P_{\mathrm{a}}=10 \mathrm{~W}$ at constant $S_{\mathrm{v}}=1,500 \mathrm{RPM}$. The following contributions of the ultrasound to the increase in $C_{\mathrm{L}}$ may be presumed; ;-5) i) A diffusion velocity of creatinine molecule increases with the relative velocity of the molecular particle which is increased by the repetitional sound pressure; ii) The thickness of concentration boundary layer on the inner surface of membrane of the tube is decreased by exchanging the solution in the neighborhood of the membrane to that at the central part in the tube by the ultrasonic cavitation and microstream; iii) The thickness of concentration boundary layer on the outer surface of the membrane is decreased by exchanging the water in the neighborhood of the membrane with that in the bulk by the ultrasonic straight flow; iv) The thickness of concentration boundary layer on the outer surface of the membrane is decreased by the similar exchange of the water to iii) by the ultrasonic cavitation and microstream; and so on.

From Eqs. (2) and (12) the following equation is given;

$$
-\ln \left(C_{1} / C_{0}\right)=-\ln \left\{1-\left(C_{\mathrm{L}} / Q\right)\right\}=K V / Q .
$$

Equation (14) represents a linear relation of $-\ln \left(C_{1} / C_{0}\right)$ with $1 / Q$ and has an intercept of $-\ln \left(C_{1} / C_{0}\right)=0$. The points of $-\ln \left(C_{1} / C_{0}\right)$ obtained from the experimental values increased linearly with $1 / Q$ but the intercept shifted slightly upward from $-\ln \left(C_{1} / C_{0}\right)=0$ as shown in Fig. 6 .

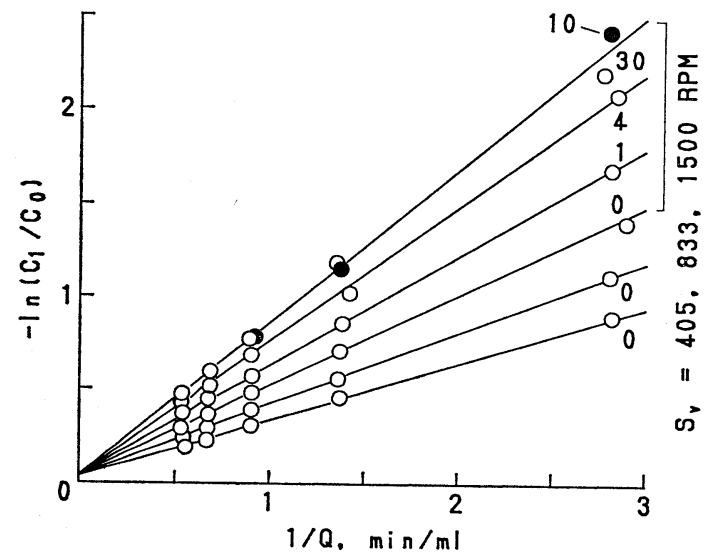

Fig. 6 The changes in $-\ln \left(C_{1} / C_{0}\right)$ with $1 / Q$ at various $S_{\mathrm{v}}$ and $P_{\mathrm{a}}(L=16 \mathrm{~cm})$. The numbers in this figure are $P_{\mathrm{a}}(\mathrm{W})$.
The increase in the inner diameter of the tube with $Q$, the non-uniform distribution of concentration of creatinine, and so on in the extremely large $Q$ may be presumed as causes of the intercept of $-\ln \left(C_{1} / C_{0}\right) \neq$ 0 , but the data are insufficient to evidence the cause. However, Eq. (14) is rewritten in the range of $Q$ in this experimental condition as;

$$
C_{\mathrm{L}}=Q\{1-\exp (-K V / Q)\},
$$

and $V$ is constant when $Q$ is stationary, so that $C_{\mathrm{L}}$ depends on $K$. Hence, the contributions of ultrasound to the enhancement of $C_{\mathrm{L}}, \mathrm{i}-\mathrm{iv}$ ), may be examined by the value of $K$ or $k_{1}$ in Eq. (15).

\subsection{Examinations of Contributions of Ultrasound to Enhancement of $C_{\mathrm{L}}$.}

\subsubsection{Examination of contribution i)}

The slope of the straight line increased with the ultrasonic power, $P_{\mathrm{a}}$, and reached the maximum position at $P_{\mathrm{a}}=10 \mathrm{~W}$ at constant $S_{\mathrm{v}}=1,500 \mathrm{RPM}$, as shown in Fig. 6. The diffusion coefficient is proportional to the relative velocity of solute molecule which is almost proportional to the amplitude of ultrasound. The slope of the line, $K V$, therefore, must increase with $\sqrt{P_{\mathrm{a}}}$ if i) were the cause. However, $K V$ did not increase with $\sqrt{P_{\text {a }}}$ at $P_{\mathrm{a}} \geqq 10 \mathrm{~W}$. This fact means that the contribution i) may not be relevant to the explanation of this experimental result.

\subsubsection{Examination of contribution ii)}

The thickness of the concentration boundary layer on the inner surface of the tube membrane related to $k_{\text {in }}$ is considered here. On the assumption that the concentration boundary layer is thinner than that of the velocity, the theoretical calculation on the inlet pipe flow can be applied. The growth length, $x_{\mathrm{i}}$, in axial direction where a fully developed Poiseuille flow is attained is shown as

$$
\frac{x_{\mathrm{i}} \nu}{Y_{1}^{2} U}=\text { constant }
$$

where $\nu$ is a coefficient of kinematic viscosity and $U$ is an average flow velocity of the solution. ${ }^{6)}$ As $x_{\mathrm{i}}$ is proportional to flow volume $Q$, and the mean thickness of the layer can be considered inversely proportional to the thickness of the layer. The following similar relation with the concentration thickness is obtained;

$$
\delta_{\text {in }} \propto 1 / Q .
$$

$R_{\text {in }}$ in Eq. (3) and $1 / k_{\text {in }}$ in Eq. (4) seem to be prop- 
ortional to $\delta_{\text {in }}$. Hence,

$$
R_{\text {in }}=1 / k_{\text {in }} \propto 1 / Q .
$$

The value of $k_{\text {in }}$, therefore, must change also with $1 / Q$. However, since $K$ is independent of $1 / Q$ as can be seen in Fig. 6, $1 / k_{\text {in }}$ in Eq. (4) may be ignored under this experimental condition, and $\delta_{\text {in }}$ may be ignored. Therefore, the contribution ii) may not be relevant to the explanation of the experimental result.

\subsubsection{Examination of contribution iii)}

As revealed in the above consideration the resistance of solute transport through the tube membrane, $R$, is given as follows under this experimental condition;

$$
R=\left(1 / k_{\mathrm{M}}\right)+\left(1 / k_{\text {out }}\right) \text {. }
$$

The clearance, $C_{\mathrm{L}}$, increased with the rotational speed of screw, $S_{\mathrm{v}}$ as can be seen in Fig. 5. This fact shows that the average thickness of concentration boundary layer on the outer surface of the membrane, $\delta_{\text {out }}$, decreases with the flow speed of water, and $1 / k_{\text {out }}$ also decreases with the flow speed of water. The decrease in $1 / k_{\text {out }}$ by the ultrasonic straight flow, macrostream, of water, therefore, must also be taken into account. However, since the flow speed of the macrostream is much slower than that by the screw, this effect may scarcely contribute to the decrease in $1 / k_{\text {out }}$. Hence the contribution iii) may not be relevant to the explanation of the experimental result.

\subsubsection{Examination of contribution iv)}

The outer surface of the 20 tubes may be studded with dead water regions due to the existence of narrow space between the tubes and other fluid mechanical contributions. The area of dead water region generally increases with the flow speed of fluid. Since the dead water regions exist on the outer surface of the membrane, the average thickness of the outer concentration boundary layer, $\delta_{\text {out }}$, dose not seem to be 0 . The dead water regions may be removed by a mechanical action of the ultrasonic cavitation and microsteam. In this experiment the slope of the line in Fig. 6 did not increase with $\sqrt{P_{\mathrm{a}}}$ at $P_{\mathrm{a}} \geqq 10 \mathrm{~W}$, so that dead water regions were removed by the ultrasonic mechanical action at $P_{\mathrm{a}}=10 \mathrm{~W}$, and $\delta_{\text {out }}$ may be about 0 . Hence, $R$ may be given here by

$$
R=1 / k_{\mathrm{M}} .
$$

The increase in $C_{\mathrm{L}}$ by ultrasonic irradiation may lead to the effective exchange of the water in the neighborhood of the outer surface of the membrane with that in the bulk by ultrasonic cavitation and microstream. The contribution iv), therefore, is the most reasonable for explaining the experimental result.

\subsection{Discussion on $k_{\mathrm{M}}$}

The value of $K_{\mathrm{M}}$, the rate constant in the membrane, was calculated to be $8.1 \mathrm{~min}^{-1}$ from the slope of line, $K V$, at $P_{\mathrm{a}}=10 \mathrm{~W}$ in Fig. 6. The diffusion coefficient of the solute molecule in the membrane, $D_{\mathrm{M}}$, is related with $K_{\mathrm{M}}$ and $k_{\mathrm{M}}$ as follows;

$$
D_{\mathrm{M}}=K_{\mathrm{M}} Y_{1} \delta_{\mathrm{M}} / 2=k_{\mathrm{M}} \delta_{\mathrm{M}} \text {. }
$$

The values $K_{\mathrm{M}}=1.4 \times 10^{-1} \mathrm{~s}^{-1}, \quad Y_{1}=100 \times 10^{-4} \mathrm{~cm}$, and $\delta_{\mathrm{M}}=11 \times 10^{-4} \mathrm{~cm}$ are substituted in Eq. (21). $D_{\mathrm{M}}$ is estimated to be $7.7 \times 10^{-7} \mathrm{~cm}^{2} / \mathrm{s}$, which is about $1 / 10$ of that of the diffusion coefficient of creatinine in water, $7 \times 10^{-6} \mathrm{~cm}^{2} / \mathrm{s}$, obtained by the empirical formula. ${ }^{7)}$ The magnitude of $D_{\mathrm{M}}$ value may be reasonable, if the free microspace in the membrane medium of the tube is presumed to be about $1 / 10$ of that in water. The proportion of the volume occupied by free space in the membrane mediums, about $1 / 10$, may be the reasonable value as judged by a steric structure of molecule of hollow fiber, cellose, swelled in water. On the other hand, the value of $D_{\mathrm{M}}$ calculated by unsteady axisymmetric diffusion equations was $1.1 \times 10^{-6} \mathrm{~cm}^{2} / \mathrm{s}^{\dagger}$, which is not so different from the above obtained value.

\subsection{Discussion of the Experimental Results in $L=4$} $\mathrm{cm}$.

The bundle of the tubes of $L=16 \mathrm{~cm}$ is slightly complicated in shape compared with the ideal straight tube as can be seen in Fig. 4, so that the liquid flows both in the inside and outside of the tube are also complex. Since it is not somewhat certain whether the complex flow affected the value of $D_{\mathrm{M}}$

† Extended calculation from Ref. 8); the simultaneous equations of the concentration of each solute diffusing radially in the dialyzer tube in the flow of the solution and in the dialyzer membrane in the still solution (the value of diffusion coefficient $D_{\mathrm{M}}$ is assumed) are solved by giving the boundary conditions of concentration at the inlet and the outlet, and the outside of the tube. The curves of the clearance $C_{\mathrm{L}}$ to best fit the experimental curve are obtained by adjusting $D_{\mathrm{M}}$. In this way the value $D_{\mathrm{M}}$ is specified. 


\section{N. TATSUMOTO et al:: ULTRASONIC EFFECT ON CLEARANCE IN DIALYZER-MODEL}

calculated by the theoretical equation for a straight tube or not, the experiment similar to the above was made by using the simpler bundle of the tubes of $L=4 \mathrm{~cm}$ at $S_{\mathrm{v}}=1,500 \mathrm{RPM}$. The shape of the bundle of the tubes of $L=4 \mathrm{~cm}$ is almost linear in the effective part of dialysis as shown in Fig. 4. The experimental values of $C_{\mathrm{L}}$ changed with $Q$ as shown in Fig. 5 in the same manner as in those $L=16 \mathrm{~cm}$. The position of curve of $C_{\mathrm{L}}$ vs $Q$ in $L=4 \mathrm{~cm}$ reached the maximum at $P_{\mathrm{a}}=15 \mathrm{~W}$, in contrast with that in $L=16 \mathrm{~cm}$. This may be considered to be due to the heterogeneous intensity of sound in the beaker (2) in Fig. 3. The values of $K_{\mathrm{M}}$ and $D_{\mathrm{M}}$ were calculated to be $1.3 \times 10^{-1} \mathrm{~s}^{-1}$ and $7.2 \times 10^{-7} \mathrm{~cm}^{2} / \mathrm{s}$, respectively, which were almost equal to those in $L=16 \mathrm{~cm}$, from the slope of line, $K V$, which were obtained by plotting $-\ln \left(C_{1} / C_{0}\right)$ vs $1 / Q$ in $P_{\mathrm{a}}=$ $15 \mathrm{~W}$. These values indicate that the theoretical values are scarcely affected by changing the shape of the bundle of the tubes, here.

\section{CONCLUSION}

As mentioned above, it was clarified that dialysate, water, could be exchanged effectively by the ultrasonic mechanical actions, cavitation and microstream, and then the efficiency of dialysis increased. However, ultrasonic irradiation can not be applied readily to the medical dialyzer, because many tissues which may be destroyed by ultrasonic irradiation exist in blood. The investigations, therefore, are expected to be made regarding the effect of ultrasonic irradiation on tissues in blood. In any event, since a limitation exists in the exchange of dialysate by mechanical stirring, it is very useful to enhance the efficiency of dialysis by ultrasonic irradiation.

\section{ACKNOWLEDGMENTS}

The authors are grateful to Prof. K. Yoneda, Medical College of Oita, for reviewing and refining the manuscript. Special thanks to Asahi Medical Co. Ltd., for supplying the hollow fiber tubes for dialysis and its data, and also to Cho-onpa Kogyo Co. Ltd., Tachikawa, Tokyo, and Kairiku Denpa Co. Ltd., Hiroshima, for the technical assistance.

\section{REFERENCES}

1) K. Ohta, Jinkou-Jinzou no Jissai (Nankoudou, Tokyo, Kyoto, 1980), pp. 1-73 (in Japanese).

2) F. A. Gotch, "Kinetics of hemodialysis," Artif. Org. 10, 272-281 (1986).

3) K. Negishi and K. Takagi, Cho-Onpa Gijutsu (Tokyo-daigaku Shuppankai, Tokyo, 1984), pp. 151-152 (in Japanese).

4) O. Nomoto, "Present states of the ultrasonic research (review)," Phys. Soc. Jpn. 18, 561-582 (1963) (in Japanese).

5) O. Nomoto, "Ultrasonic cavitation: Review of recent progress (1)," J. Acoust. Soc. Jpn. (J) 28, 348-356 (1972) (in Japanese).

6) H. Schlichting, Boundary-Layer Theory (McGrawHill Book Co., New York, 1979), pp. 241-242.

7) P. J. Hillson and R. B. McKay, "Aggregation of dye molecule in aqueous solution. A polarographic study," Trans. Faraday Soc. 61, 374-382 (1965).

8) J. Crank, The Mathematics of Diffusion (Oxford Univ. Press, London, 1970), pp. 62-98. 